\title{
Rod-Like Trabeculae Extraction from Cancellous Bone Microstructure using Topological Analysis
}

\author{
Xiaoting Wang* \\ Y.K. Choi ${ }^{\dagger}$ \\ W.W. Lu ${ }^{\ddagger}$ \\ Wenping Wangt \\ xtwang@sdu.edu.cn ykchoi@cs.hku.hk wwlu@hkusua.hku.hk wenping@cs.hku.hk \\ * School of Computer Science and Technology, Shandong University, China \\ t The Department of Computer Science, The University of Hong Kong, China \\ $¥$ The Department of Orthopaedics and Traumatology, The University of Hong Kong, China
}

\begin{abstract}
The study of the microstructure in cancellous bone is important in osteoporosis research. In this paper, we focus in the extraction of rod-like trabeculae in cancellous bone, whose topological and geometrical properties are known to be strongly related to bone strength. Three dimensional (3D) cancellous bone volume data obtained from micro Computed Tomography (microCT) scans is used in our analysis. Because of the complexity of the cancellous bone microstructure, the mere use of existing $3 D$ digital topology methods is unable to yield satisfactory results for trabeculae extraction. We devise several enhancements which are specific to the identification of rod-like trabeculae. Experimental results show that the accuracy of trabeculae extraction is greatly improved. This application study may help clinicians to solve problems in future.
\end{abstract}

\section{Introduction}

Osteoporosis is mainly due to bone mass loss and bone tissue deterioration and has adverse effects to bone strength. Patients with osteoporosis have higher risk of bone fracture. Researches show that, apart from bone mineral density, the degree of osteoporosis is also determined by the properties of bone microstructure. Cancellous bone, also known as spongy or trabecular bone, is highly porous and contains a network of bone strands called trabeculae. There are rod-like and plate-like trabeculae, the ratio of these two kinds provides a basic insight to the structural property of the cancellous bone. Bone strength and other mechanical properties are also found to be largely correlated to the topological and geometrical properties of the trabeculae [2]. Therefore, finite element models of cancellous bone are often built to study these properties [10].

Three dimensional (3D) data of cancellous bone is mainly obtained using micro-CT scans which give a much higher image resolution than traditional CT imaging. Various image processing techniques are needed to enhance the datasets before a thorough analysis can be made. It is essential that these techniques are topology preserving if the topological properties of the scanned objects are the subject of studies. In this case, digital topology [7] provides a mathematical tool to facilitate the solving of topological problems for 3D volumetric datasets. Saha et. al. devised a topological characterization method to classify each bone voxel as a curve, surface or other element types [13]. From this classification, results such as surface-to-curve ratio, erosion index, etc., can then be calculated which will provide references to the structural properties of the cancellous bone. Based on the same techniques in digital topology analysis, Gomberg et al. further determined the orientation of plate-like trabeculae by apply appropriate measures to voxels that are classified as surface type [4].

The thickness, the connectivity, and the number of rod-like trabeculae give us quantifiable information in evaluating osteoporosis. In such cases, apart from voxelby-voxel classification, each rod should be extracted as an entity for further analysis. There are various segmentation algorithms that work best depending on the different nature of the volume dataset and the applications behind. Thresholding is a basic and simple method [15]. Some use the structural information of an image to detect the edge and find the boundary of a region [1][16]. Morphological techniques [14] and region growing [5] are also used in obtaining segmented regions. Isosurfaces [8], energy minimization active contours [6] and level sets [9] are popular techniques in medical imaging segmentation. In this paper, we devise a method for extracting rod-like trabeculae in cancellous bone based on Saha et al.'s work [13], utilizing the classification results obtained in the previous stage. The identification of rod-like trabeculae is more difficult due to their relatively smaller structural size than plate-like trabeculae. Various refinements are therefore needed to give better extraction results. 
In section 2, we will describe the basic steps of applying general digital topology algorithms in trabeculae extraction. The three enhancements designed specifically for the extraction of rod-like trabeculae and their results will be presented in details in section 3. Finally, we will conclude the paper in section 4 .

\section{Rod-like trabeculae extraction}

Our primary aim is to extract the rod-like trabeculae given a 3D dataset $B$ of a piece of cancellous bone. The basic steps are outlined as follows:

1. Binarize $B$ by choosing an appropriate threshold and obtain its skeleton $B_{s}$ by a thinning procedure that preserves the topology of the bone.

2. Classify the voxels in $B_{s}$ to different topological types.

3. A curve in $B_{s}$ consisting of a connected sequence of curve-type voxels longer than a preset threshold is treated as a rod-like trabecula.

4. The identified thinned rod-like structures are further expanded to include neighbouring voxels in the original volume $B$, so that the corresponding rods-like trabeculae in the original dataset are restored.

\subsection{Thresholding and Thinning}

A volume data contains voxels with scalar values representing certain properties, e.g. bone density for a bone dataset. These values may vary in a wide range and as in digital image processing, it is often more desirable to work with binary values in the subsequent manipulations. Each voxel is assigned a binary value 0 or 1 according to a threshold. It is important to select a threshold such that the connectivity of the data is preserved after the binarization [3]. We then perform a thinning operation proposed by Saha et al. [12] which is topology preserving and uses local topological information. A thick surface in the dataset will then become a slim surface of 1-voxel thickness while a thick pillar will be changed into a 1voxel thick curve (Fig. 1). The thinning process consists of a number of iterations, each of which is to remove a shell of outermost voxels from the bone structures. The removal of voxels is done in a topology preserving manner, meaning that the topological properties of the bone structures before and after voxel removal are the same. Using the thinned dataset, one can work with a much simpler representation of the cancellous bone structure while retaining the original shape information. The skeletonized cancellous bone dataset will then be used in the following classification phase.

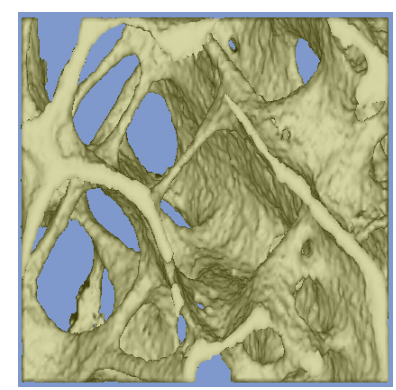

(a)

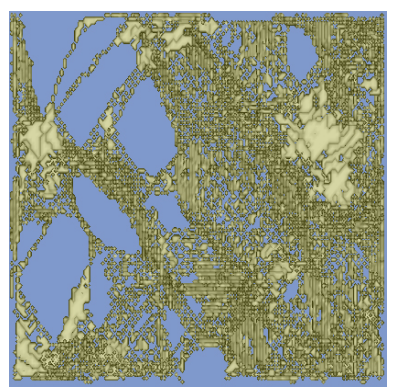

(b)
Figure 1: (a) Original cancellous bone data; (b) thinned bone data

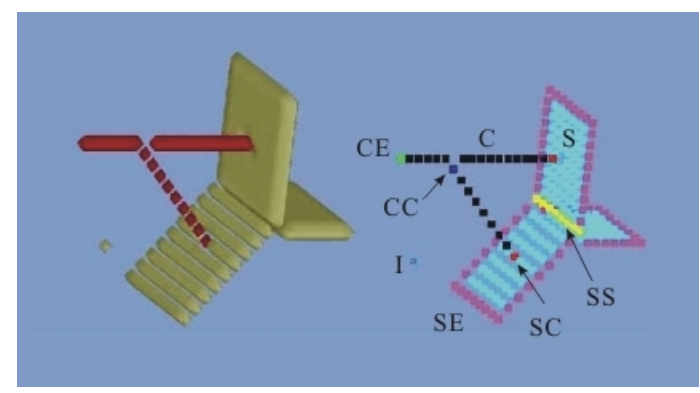

Figure 2: (Left) A thinned synthesized dataset; (Right) Each voxel is classified into different topological types, represented by different colours.

\subsection{Classification}

We now need to differentiate each bone voxel in the skeletonized volume according to their topological properties. We apply a classification scheme that assigns a topological type to each bone voxel [13]. After this step, each bone voxel is categorized into one of topological types (Fig. 2): I-type (isolated), C-type (curve), CE-type (curve edge), S-type (surface), SE-type (surface edge), CC-type (curve-curve joint), SS-type (surface-surface joint) or SC-type (surface-curve joint). The classification gives us useful information as to how each voxel contributes to the shape of the cancellous bone. Fig. 2 illustrates the classification of a synthesized dataset. As the skeleton of a rod-like trabecula resembles a curve, we are most interested in those voxels that are classified as Ctype. Next, we will describe how a curve composed of connected C-type voxels can be obtained in its entirety.

\subsection{Segmentation}

After the thinning and the classification operations, every bone voxel in the skeletonized volume is associated with a topological type. With the type information, 

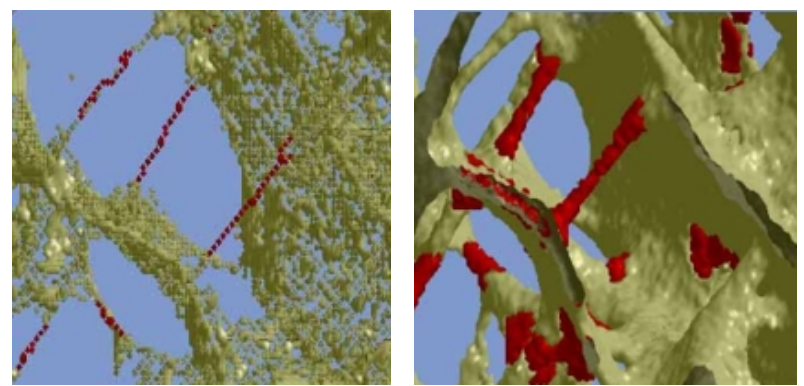

Figure 3: (Left) Rod-like structures identified in the thinned dataset (those marked in red); (Right) The corresponding rod-like trabeculae in the original dataset.

segmentation operation can then be applied and the rodlike trabeculae can be identified. There are some voxels being classified as CC-, SS-, SC-types, which may be treated as the junctions between curves and surfaces. The segmentation can therefore be done by removing these junction voxels together with their 26-connected neighbours, and disjoint structures could then be obtained [11]. Since rod-like trabeculae in general have a minimum span, only those structures containing entirely of C-type voxels in the thinned volume which are longer than a specific length will be identified.

\subsection{Rod-like trabeculae restoration}

At this stage, the rod-like trabeculae are identified in the thinned volume; the next step is then to restore the corresponding trabeculae in the original dataset. Recall that in the thinning stage, the outermost voxels are removed layer by layer. We there associate with each removed voxel a number which is the iteration in which it is removed by the thinning algorithm. A larger iteration number implies that the corresponding voxel is removed in a later iteration. Therefore, we may carry out a reversal of the thinning process, that is, to grow the identified thinned structure gradually to include neighbouring voxels, first with those having maximum iteration number and then with those having smaller ones. The final results would be a set of voxels in the original volume that are classified as rod-like trabeculae (Fig. 3).

\section{Extraction enhancements}

Intuitively, in the segmentation stage, curves in the skeletonized volume correspond to rod-type trabeculae and they are identified. However, due to the small size of rod-type trabeculae, it is often difficult to obtain satisfactory results using only this simple criterion. In fact, extraction results have both false negatives where rod-type trabeculae are not found, as well as false positives where regions not containing rod-type trabeculae are identified. In this section, we will present three enhancements and also their results when applying to 8 typical cancellous bone datasets. The rod-like trabeculae are first identified by inspection as a reference set. We recognize that this can be subject to the judgment of individual medical practitioner and also the specific identification criteria for the applications in which the rod-like trabeculae are to be used; nevertheless, the numbers of false positives and false negatives are calculated according to the reference set for evaluating the performance of the enhancing methods. Table 1 shows the outcome of the extraction using the basic steps without any enhancements. The figures will be used to compare with the outcome of each of the enhancements discussed below.

Table 1. Extraction outcome using basic steps without any enhancement.

\begin{tabular}{|l|l|c|c|}
\hline & $\begin{array}{l}\text { Number of } \\
\text { rod-like } \\
\text { trabeculae } \\
\text { identified by } \\
\text { the basic steps }\end{array}$ & $\begin{array}{l}\text { Number of } \\
\text { false positives } \\
\text { resulted from } \\
\text { the basic steps }\end{array}$ & $\begin{array}{l}\text { Number of } \\
\text { false negatives } \\
\text { resulted from } \\
\text { the basic steps }\end{array}$ \\
\hline Set 1 & 100 & 72 & 8 \\
\hline Set 2 & 75 & 45 & 6 \\
\hline Set 3 & 85 & 52 & 8 \\
\hline Set 4 & 62 & 50 & 7 \\
\hline Set 5 & 86 & 62 & 12 \\
\hline Set 6 & 56 & 40 & 2 \\
\hline Set 7 & 41 & 36 & 4 \\
\hline Set 8 & 72 & 55 & 11 \\
\hline
\end{tabular}

\subsection{Major direction determination}

Some false positives are found to be lying on platelike trabeculae of the bone. A closer look into the corresponding thinned structures reveals that each of these thinned structures is composed of some scattered voxels that happen to be a connected set of C-type voxels. These scattered voxels, however, do not possess a major direction in their positional arrangements, whereas such a direction is often quite obvious for the voxels of rod-like trabeculae. Therefore, an extra restriction is imposed, namely, only those sets of C-type voxels that possess a major direction are identified. To determine whether a major direction exists for a set of voxels, we shall make use of a covariance matrix. 

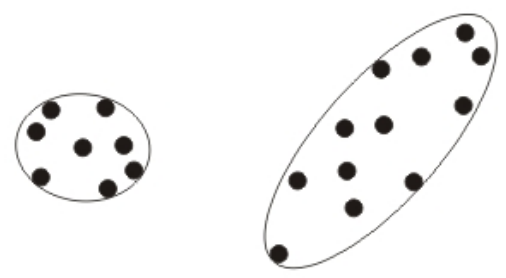

Figure 4: A 2D illustration showing two clusters of voxels enclosed by ellipsoids. The cluster on the right possesses a major direction which is along the longest axes of the ellipsoid.

Given a set of $n$ voxels $P_{i}=\left(a_{i}, b_{i}, c_{i}\right)^{\mathrm{T}}, i=1, \ldots, n$. Let $M$ be the mean of a cluster of voxels, i.e. $M=\sum P_{i} / n$ and $Q_{i}=P_{i}-M=\left(x_{i}, y_{i}, z_{i}\right)^{T}$ be the directional vectors

to each voxel $P_{i}$ from $M$. The covariance matrix of the voxels is defined by

$$
S=\left(\begin{array}{llll}
Q_{1} & Q_{2} & \ldots & Q_{n}
\end{array}\right)\left(\begin{array}{c}
Q_{1}^{T} \\
Q_{2}^{T} \\
\vdots \\
Q_{n}^{T}
\end{array}\right)=\left(\begin{array}{ccc}
\sum x_{i}^{2} & \sum x_{i} y_{i} & \sum x_{i} z_{i} \\
\sum x_{i} y_{i} & \sum y_{i}^{2} & \sum y_{i} z_{i} \\
\sum x_{i} z_{i} & \sum y_{i} z_{i} & \sum z_{i}^{2}
\end{array}\right)
$$

The covariance matrix $S$ is a real symmetric matrix and therefore has three real eigenvalues. The matrix $S$ is also either positive definite or positive semi-definite, meaning that the eigenvalues are non-negative. In fact, the three eigenvalues and their corresponding eigenvectors give an ellipsoid (can be a degenerate one) centred at $M$ containing the $n$ voxels; the length of the three axes of the ellipsoid are given by the eigenvalues while the directions of the axes are indicated by the corresponding eigenvectors (Fig. 4). If one of the eigenvalues is much larger than the other two, the ellipsoid is elongated and there must be a major direction given by the corresponding eigenvector. We may then discard those
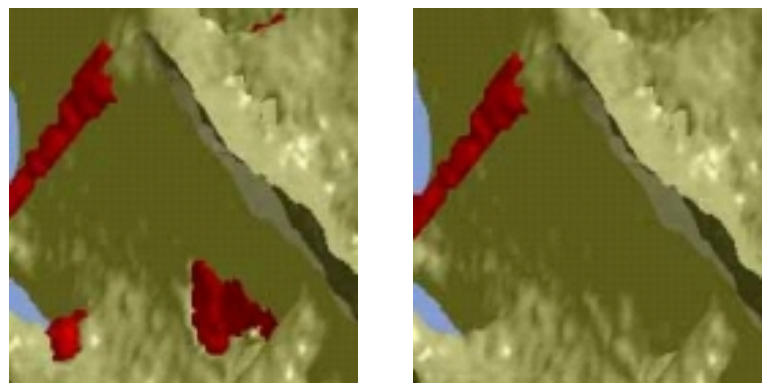

Figure 5: Extraction result (Left) before; (Right) after applying major direction determination. The identified rod-like trabeculae are marked in red. Note that the false positives on the plate-like trabeculae are discarded.
Table 2. Experimental results of using major direction determination.

\begin{tabular}{|l|l|l|}
\hline & $\begin{array}{l}\text { Number of false } \\
\text { positives resulted from } \\
\text { the basic steps }\end{array}$ & $\begin{array}{l}\text { Number of false } \\
\text { positives eliminated by } \\
\text { major direction } \\
\text { determination }\end{array}$ \\
\hline Set 1 & 72 & 59 \\
\hline Set 2 & 45 & 37 \\
\hline Set 3 & 52 & 43 \\
\hline Set 4 & 50 & 44 \\
\hline Set 5 & 62 & 46 \\
\hline Set 6 & 40 & 30 \\
\hline Set 7 & 36 & 29 \\
\hline Set 8 & 55 & 41 \\
\hline
\end{tabular}

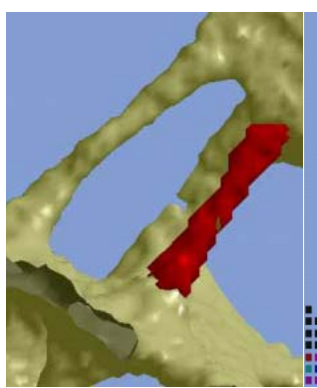

(a)

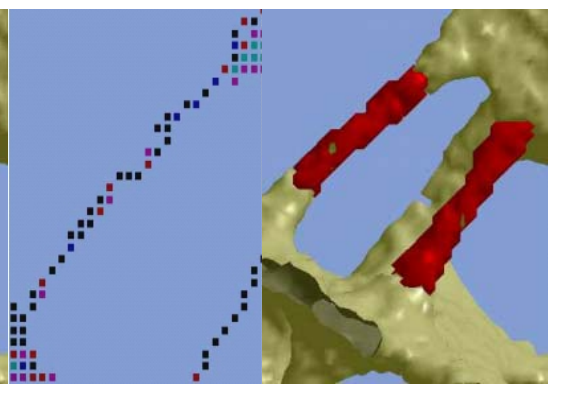

(b)

(c)
Figure 6: (a) A rod-like trabecula (the leftmost one) is missed; (b) the classification of voxels shows that the missed trabecula is formed by short curve segments (in black) connected by curve-curve junctions (in red); (c) it is recovered after applying short curves combination.

curve segments that do not possess a major direction and this can greatly reduce the number of false positives as shown by our experiments (Fig. 5).

Table 2 gives the results of rod-like trabeculae extraction from the same 8 typical datasets of cancellous bone as in Table 1. The second column shows the number of incorrect identification of rod-like trabeculae by the basic steps without any enhancement, while the third column shows the number of such false positives removed by determining the major direction of extracted curve segments.

\subsection{Short curves combination}

In the segmentation process, dismantled structures are treated as rod-like trabeculae only if their lengths are larger than a user defined threshold. This restriction is to ensure that tiny curve segments would not be identified. However, there are still some rod-like trabeculae longer than the threshold but not extracted (Fig. 6a). By studying 
Table 3. Experimental results of using short curves combination.

\begin{tabular}{|l|c|c|}
\hline & $\begin{array}{l}\text { Number of false } \\
\text { negatives resulted } \\
\text { from the basic steps }\end{array}$ & $\begin{array}{l}\text { Number of rod-like } \\
\text { trabeculae captured by } \\
\text { short curves combination }\end{array}$ \\
\hline Set 1 & 8 & 6 \\
\hline Set 2 & 6 & 5 \\
\hline Set 3 & 8 & 6 \\
\hline Set 4 & 7 & 6 \\
\hline Set 5 & 12 & 10 \\
\hline Set 6 & 2 & 1 \\
\hline Set 7 & 4 & 3 \\
\hline Set 8 & 11 & 10 \\
\hline
\end{tabular}

Table 4. Experimental results of applying thin surface recognition.

\begin{tabular}{|l|c|c|}
\hline & $\begin{array}{l}\text { Number of false } \\
\text { negatives resulted } \\
\text { from the basic steps }\end{array}$ & $\begin{array}{l}\text { Number of rod-like } \\
\text { trabeculae captured by } \\
\text { thin surface recognition }\end{array}$ \\
\hline Set 1 & 8 & 0 \\
\hline Set 2 & 6 & 0 \\
\hline Set 3 & 8 & 1 \\
\hline Set 4 & 7 & 1 \\
\hline Set 5 & 12 & 1 \\
\hline Set 6 & 2 & 0 \\
\hline Set 7 & 4 & 1 \\
\hline Set 8 & 11 & 0 \\
\hline
\end{tabular}

Table 5. Outcome of rod-like trabeculae extraction with enhancements.

\begin{tabular}{|c|c|c|c|}
\hline & $\begin{array}{l}\text { Number of rod- } \\
\text { like trabeculae } \\
\text { identified }\end{array}$ & $\begin{array}{l}\text { Number of } \\
\text { false positives }\end{array}$ & $\begin{array}{l}\text { Number of } \\
\text { false negatives }\end{array}$ \\
\hline Set 1 & 47 & 13 & 2 \\
\hline Set 2 & 43 & 8 & 1 \\
\hline Set 3 & 49 & 9 & 1 \\
\hline Set 4 & 25 & 6 & 0 \\
\hline Set 5 & 51 & 16 & 1 \\
\hline Set 6 & 27 & 10 & 1 \\
\hline Set 7 & 16 & 7 & 0 \\
\hline Set 8 & 41 & 14 & 1 \\
\hline
\end{tabular}

the thinned structure and the classification of voxels of these false negatives, it is found that their structures may contain several short curve segments connected by CCtype voxels. This kind of structures will be excluded by the extraction process due to the short length of individual curve segments. In view of this, the following steps are applied in attempt to combine all the short curve segments that appear to be on the same rod-like trabecula:

1. In the segmentation stage, all curve segments shorter than the user defined threshold are stored.
2. These short curve segments are grouped into clusters based on their geometric proximity.

3. Each cluster of short curve segments is then subject to the major direction determination, and is classified as a rod-like trabecula if its voxels possess a major direction.

Applying the short curves combination enhancement can discover some of the missed rod-like trabeculae (Fig. 6) and the experimental results are shown in Table 3, where the third column shows the number of rod-like trabeculae thus reclaimed.

\subsection{Thin surface recognition}

We observe that the skeletons of rod-like trabeculae may not necessarily be curve segments, but rather might appear as thin strips and therefore be classified as surfaces; these are again ignored by the extraction process. In order to capture these missing structures, for each segmented part which is classified as a surface, we will determine whether it is a thin strip. Let $w$ be the user defined value which is the maximum width of a strip that can be accepted as a rod-like trabecula. To decide whether a cluster of voxels $S$ classified as a surface should be taken as rod-like, let $C$ be the centre of the set of voxels in $S$ and consider a sphere $I$ of radius $w / 2$ centred at $C$. If the set of voxels $S^{\prime}=S-I$ is not 26-connected, we say that $S$ is a thin strip and will be identified as rod-like trabeculae.

However, it can be time-consuming to determine the connectivity of $S$, as it may contain many voxels. To improve the efficiency, we reduce the set $S$, by considering only those voxels contained within another sphere $O$ also centered at $C$ whose radius is greater than $w / 2$, i.e. $S^{\prime}=O-I$. In other words, we will only check the connectivity of voxels contained in between the outer sphere $O$ and the inner sphere $I$.

Although it is not very common to have rod-like structures being classified as surface type, this situation indeed exists. Table 4 shows the results of applying thin surface recognition.

\subsection{Overall results}

After applying the three enhancements discussed above, the numbers of false positives and false negatives are greatly improved (Table 5). The visual results of applying the enhancements are shown in Fig. 7. We remark that the identification of rod-like trabeculae is hard, which often requires the judgment of experienced practitioners that can vary among individuals. An advantage of our enhancements is that their refinements can be controlled by fine-tuning the parameters. For 
example, one may alter the value $w$, the accepted width for a thin strip, to add or remove more trabeculae to be identified. Likewise, the maximum difference between the eigenvalues which decide the shape of the ellipsoid for major direction determination is also adjustable.

\section{Conclusion}

We have presented a method for identifying rod-like trabeculae from a 3D cancellous bone dataset, which can be used by clinicians for better analysis and understanding of the datasets. Our method is based on various digital topology algorithms used in the thinning, classification and segmentation of the dataset. The results are further improved by applying three enhancements which are specifically designed for rod-like trabeculae extraction. The enhancements are parameter adjustable and therefore are well suited for practitioners to control the extraction results.

The current enhancing methods in extracting rod-like trabeculae work upon the output of the generic digital topology algorithms used in the previous stages and therefore have limitations in further improvements. One possibility is to impose criteria and restrictions specific to rod-like trabeculae extraction in earlier stages of thinning, classification and segmentation.

\section{References}

[1] J. Canny, "A computational approach to edge detection," IEEE Trans. Pattern Anal. Machine Intell., PAMI-8, 1986, pp. 679-698.

[2] R. T. DeHoff, E. H. Aigeltinger and K. R. Craig, "Experimental determination of the topological properties of three-dimensional microstructures," J Microsc 95, 1972, pp. 6991.

[3] B. R. Gomberg, P. K. Saha, H. K. Song, S. N. Hwang, and F. W. Wehrli, "Topological analysis of trabecular bone MR images," IEEE Trans. Med. Imag. 19 (3), 2000, pp. 166-174.

[4] B.R. Gomberg, P.K. Saha, and F. W. Wehrli, "Topologybased orientation analysis of trabecular bone networks," Med. Phys 30 (2), 2003, pp. 158-168.

[5] R. M. Haralick and L. G. Shapiro, "Image segmentation techniques," Computer Vision, Graphics, and Image Processing, 29(1), 1985, pp 100-132.

[6] M. Kass, A. Witkin, and D. Terzopoulos, "Snakes: active contour models," International Journal of Conputer Vision, 1(4), 1988,pp 321-331.

[7] T. Y. Kong and A. Rosenfeld, "Digital topology: introduction and survey," Comput. Vision Graphics Image Process 48, 1989, pp. 357-393.

[8] W.E. Lorensen and H.E. Cline, "Marching Cubes: a high resolution 3D surface construction algorithm," Computer

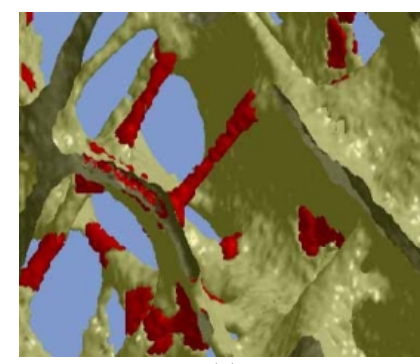

(a)

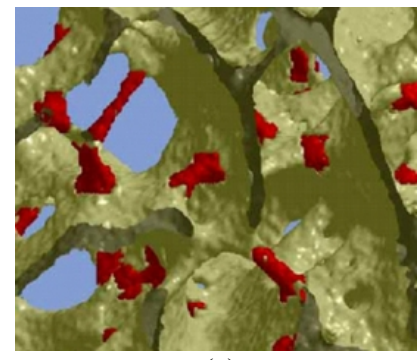

(c)

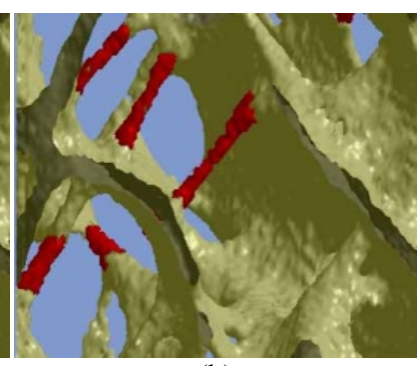

(b)

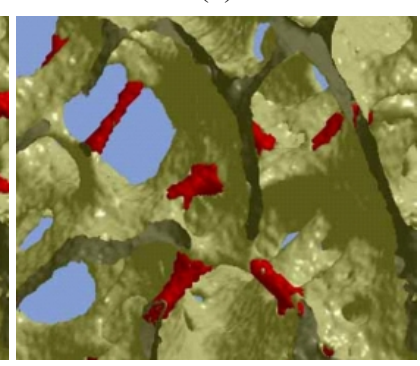

(d)

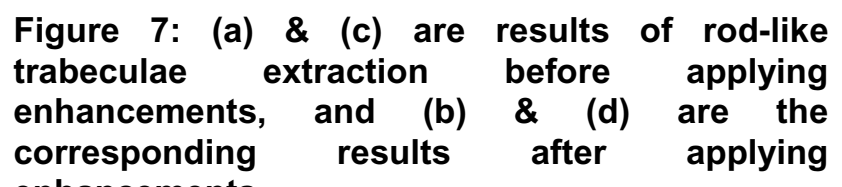
enhancements.

Graphics (SIGGRAPH '87), 21 (4), 1987, pp. 163-169.

[9] R. Malladi, J. A. Sethian, and B. C. Vemuri, "A fast level set based algorithm for topology-independent shape modeling," $J$. Math, Imaging and Vision, 6(2/3), 1996, pp 269-290.

[10] L. Pothuaud, B. van Rietbergen, C. Charlot, E. Ozhinsky, and S. Majumdar, "A new computational efficient approach for trabecular bone analysis using beam models generated with skeletonized graph technique," Comput. Meth. Biomech. Biomed. Eng. 7 (4), 2004, pp. 205-213.

[11] P. K. Saha and B. B. Chaudhuri, "3D digital topology under binary transformation with applications," Comput. Vis. Image Underst. 63, 1996, pp. 418-429.

[12] P. K. Saha, B. B. Chaudhuri, and D. D. Majumder, "A new shape preserving parallel thinning algorithm for 3D digital images," Pattern Recogn. 30, 1997, pp. 1939-1955.

[13] P. K. Saha, B. R. Gomberg, and F. W. Wehrli, "Threedimensional digital topological characterization of cancellous bone architecture," Int. J. Imaging Syst. Technol. 11, 2000, pp. 81-90.

[14] J. Serra, Image Analysis and Mathematical Morphology, Academic Press, 1982.

[15] J. S. Weszka, "A survey of thresholding techniques", Computer Graphics, and Image Processing, 7, 1978, pp 259-265.

[16] S. W. Zucker and R. A. Hummel, "A three-dimensional edge operator," IEEE Transactions on Pattern Analysis and Machine Intelligence, 1981, pp 324-331. 